\title{
SIX CASES OF TRAUMATIC RUPTURE OF THE BRONCHUS
}

\author{
BY \\ MICHAEL BATES AND HARVEY J. BEARD \\ From the North Middlesex Hospital and Kelling Hospital, Norfolk
}

(RECEIVED FOR PUBLICATION APRIL 2, 1956)

The operative repair of traumatic rupture of the bronchus, until a few years ago a matter for conjecture, has now proved successful on several occasions. Two such cases are presented here, and reports of four other cases of traumatic bronchial rupture. Together, the six cases show a variety of ways in which the condition may end ; two have had atelectatic lungs for many years, a third had a pneumonectomy within two weeks of the accident, the fourth and fifth cases have healthy, functioning lungs after late operative repair of the injured bronchus, and the sixth case appears likely to make a satisfactory spontaneous recovery.

In discussing the cases some of the literature is reviewed and certain points which are of importance in diagnosis and treatment are noted.

\section{CASE RePorts}

CASE 1.-Miss O. B., aged 26, was admitted on November 5, 1954, to the Brompton Hospital under the care of Mr. O. S. Tubbs. She complained of a cough and sputum which she had had for the past two years.

At the age of 5 years she was run over by a van and received chest and head injuries. She was unconscious for a time. She had a fracture of the right fourth rib, surgical emphysema, and a right pneumothorax. She was admitted to King's College Hospital, London, and remained there for four months. During this time she had a bronchoscopy performed by Mr. Negus and an attempt made to canalize the right main bronchus. In April, 1934, she was admitted to the Westminster Hospital, where bronchial dilatation was performed three times by Mr. Tudor Edwards. The right upper lobe was said to have re-expanded. Further bronchoscopic dilatation was performed four months later. At the age of 10 she had left-sided pleurisy and was in bed for three to four weeks; this condition recurred at the age of 18 and she was in bed for three weeks. For the last two years she had had persistent cough and sputum throughout the year; the sputum became yellow when she had a cold. The sputum had been occasionally blood-stained. As a result of a mass radiograph in 1954 she was advised to seek a surgical opinion.
On examination the patient was thin but otherwise fit. The fingers were not clubbed. Gross inspiratory stridor was present. Chest examination revealed that the trachea deviated to the right ; there was good expansion of both sides, but the left expanded better than the right. The percussion note was resonant all over the chest and there were normal breath sounds on both sides; there were no added sounds. The pulse was 80 , regular. The jugular venous pressure was not raised. Blood pressure readings in the right arm gave $115 / 80 \mathrm{~mm}$. $\mathrm{Hg}$, in the left arm $110 / 80$ $\mathrm{mm}$. Hg. The apex beat of the heart was palpable in the fifth interspace in the right axilla ; heart sounds were normal. The abdomen and central nervous system were healthy.

A chest radiograph (Fig. 1) showed the heart lying entirely in the right posterior chest. The left lung filled both sides of the chest and there was no sign of the right lung. Acid-fast bacilli were not found in the sputum, but culture gave a heavy growth of Staphylococcus aureus. Haemoglobin was $12.4 \mathrm{~g} . \%$. Nothing abnormal was found in the urine.

On November 19, 1954, bronchoscopy was performed by Mr. Tubbs. The cords and trachea were normal. There was moderate stenosis of the left main bronchus at its origin. Below this the lumen was of normal calibre, but the left upper lobe bronchus arose from the anterior wall of the main bronchus. There was a moderate amount of mucopus in the segmental branches of the lower lobe. The right main bronchus was $\frac{3}{4}$ in. long and terminated as a transverse pocket. No sign of an opening beyond this could be demonstrated.

Bronchograms (Figs. 2, 3, 4) were performed and revealed a moderate stenosis of the origin of the left main bronchus and a short, completely closed pocket which represented the right main bronchus. The left upper lobe had swung across to fill the right chest and the left apical lower filled the upper half of the left chest, the lower half being occupied by the left basal segments.

Respiratory function tests were performed and gave: M.B.C., $44.4 \mathrm{l}$./min., B.R., $36.3 \mathrm{l}$./min., V.C., $2,650 \mathrm{ml}$., M.E. $63.6 \%$.

The patient was admitted with the object of determining whether the cough and sputum were arising from the remnant of the right lung, assuming that this had a bronchial communication. It was clear that the right main bronchus was completely closed off 

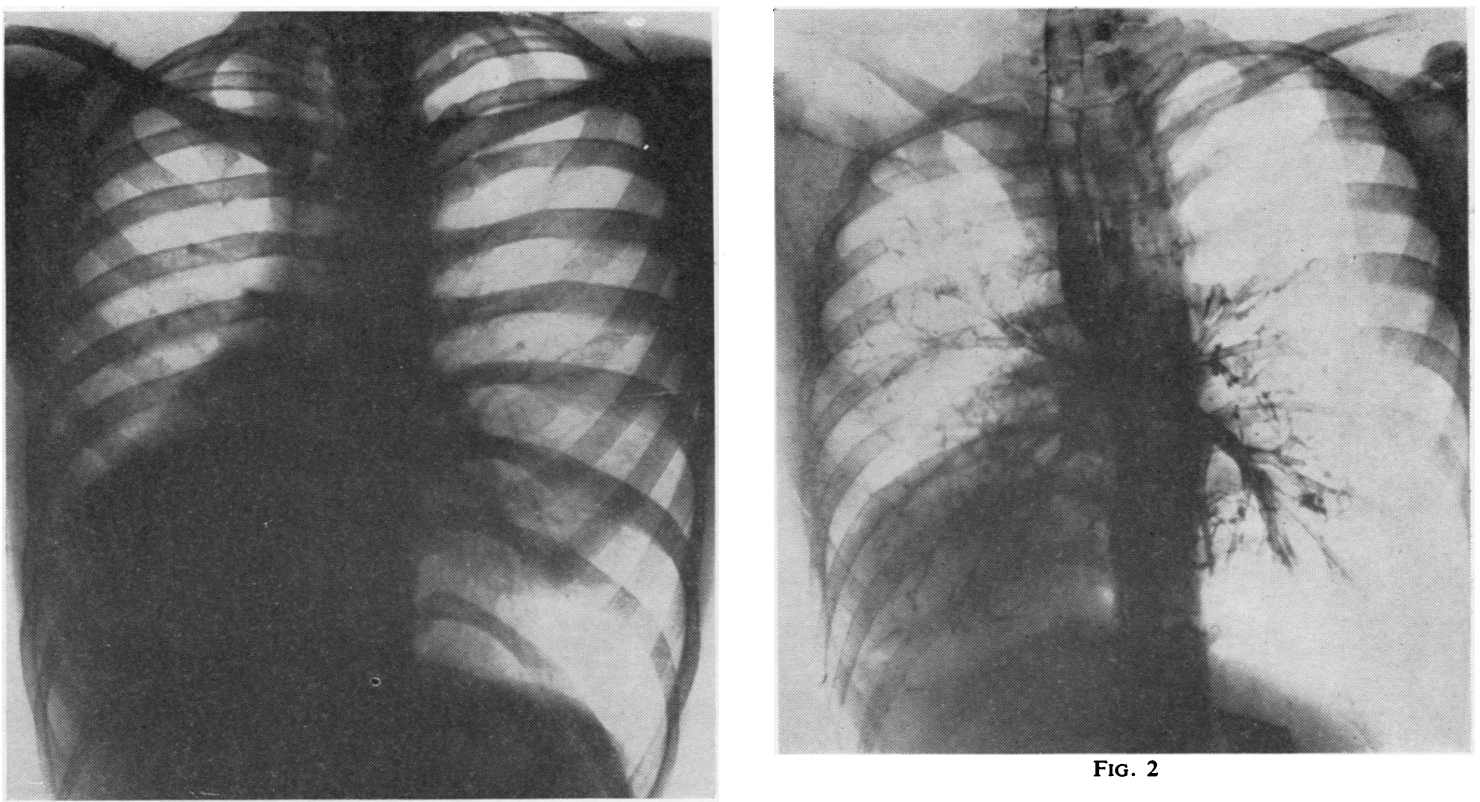

FIG. 2

FiG. 1.-Case 1: Radiograph taken in April, 1954.
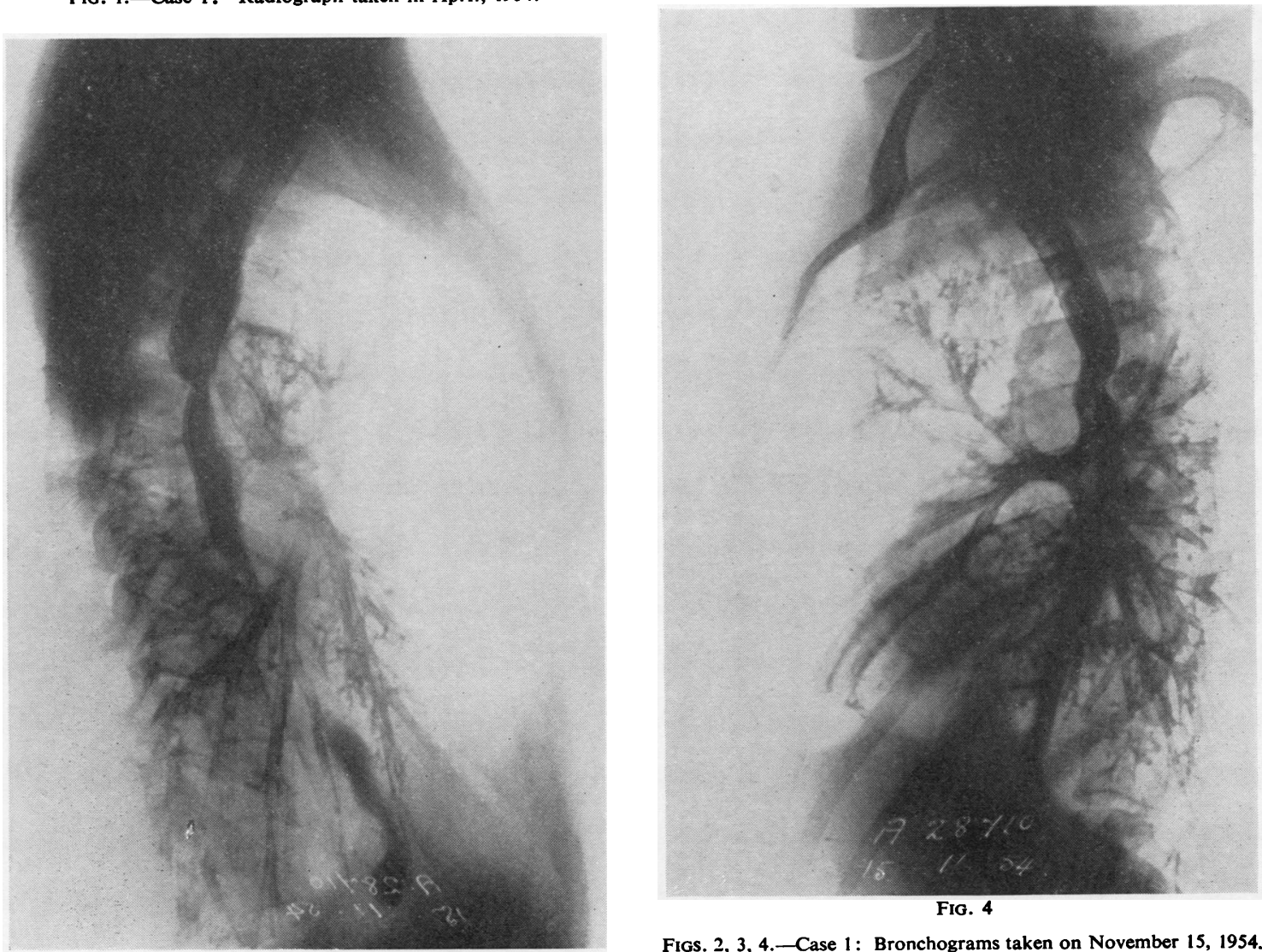

Fig. 4

Figs. 2, 3, 4.-Case 1: Bronchograms taken on November 15, 1954.

Fig. 3 
and that the diagnosis was therefore one of mild chronic bronchitis of the left lung, for which no treatment was at present indicated.

CASE 2.-G. M., a girl aged 14, was admitted in January, 1954, to Warwick Hospital under the care of Mr. R. Abbey Smith. Her history was that in 1944 at the age of 3 she was run over by a cart, one of the wheels passing over her chest and neck. Unfortunately radiographs taken at that time have been destroyed. Ever since then she had had a cough, especially in the mornings, with a small amount of sputum which was mucopurulent but never fetid or blood-stained. Following head colds, the chest symptoms increased, but she had lost very little time from school and appeared to have had a fairly normal childhood. There was no history of attacks of pneumonia or pleurisy.

She was originally referred to Dr. Edgar Moyes by her general practitioner, who had found the apex beat and trachea well over to the left and could not explain his clinical findings.

She was a well-built girl of normal size for her age, with normal chest development. The radiograph (Fig. 5) showed little evidence of contraction of the left hemithorax. A Mantoux test was positive.

Bronchoscopy on January 3, 1954, showed the left main bronchus almost totally occluded, but with a small meatus through which thick yellow pus exuded when the patient coughed. The sputum was negative for acid-fast bacilli.

A right bronchogram (Fig. 6) showed a normal right bronchial tree and a completely occluded left main bronchus.

The following notes were obtained from the original case history after the accident, which occurred on April 6, 1944. Five weeks after the accident, on May 14, 1944, bronchoscopy was performed under

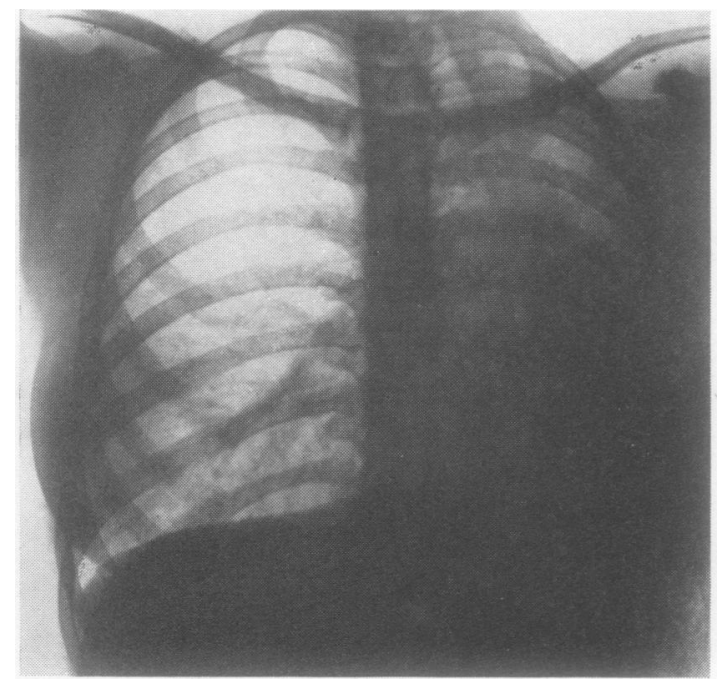

FIG. 5.-Case 2: Radiograph taken in September, 1953.

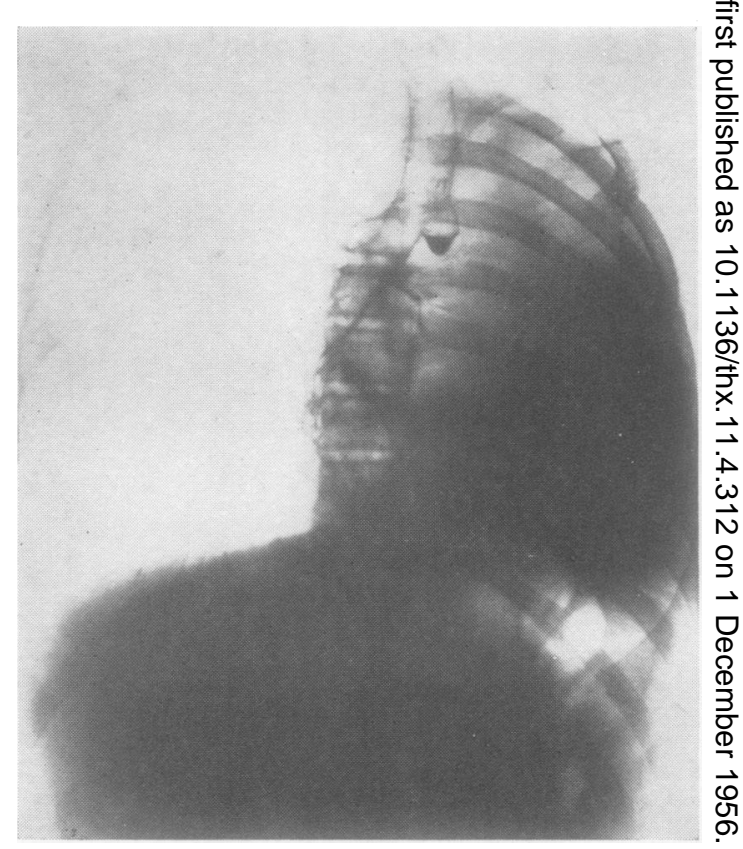

Fig. 6.--Case 2: Bronchogram January, 1954, showing complete obstruc:ion of the left main bronchus.

general anaesthesia, and showed the right bronchus to be normal; the left main bronchus was completely occluded by a plug of mucopus. When this was $\frac{1}{\Phi}$ aspirated the mucous membrane was seen to be very oedematous and almost blocking the lumen. On 음 June 2, 1944, bronchoscopy under general anaesthesia 3 was repeated and showed the left main bronchus still inflamed and oedematous but with much less mucopus.

CASE 3.-K. H., a boy aged 7 years, on January 12 , 1952, was playing on a farm when a heavy tractor 음 wheel fell on him. The child was first sent to the $\underset{x}{x}$ local hospital, where he was given $\frac{1}{8}$ grain morphia. He was then admitted to the Leicester Royal Infirmary under the care of Mr. Gordon Cruickshank. On admission at 6.30 p.m. the patient was very shocked 3 and cyanosed (pulse 100, blood pressure 95/60 $\mathrm{mm}$. Hg). The upper chest was bruised and there were closed fractures of the left femoral shaft and $D$ left clavicle. The chest showed signs of tension pneumothorax on the left side, and there were only $\bar{N}$ very faint breath sounds on the right side. There was some guarding of the abdomen. The chest radio- $\mathrm{O}$ graph (Fig. 7) showed a tension pneumothorax on the left and a moderate pneumothorax on the right side. $\sigma$

$A$ blood transfusion was given and the patient placed in an oxygen tent, when $800 \mathrm{ml}$. air was aspirated from the left chest, the pressure before this $\mathbb{D}$ being plus $25 \mathrm{ml}$. water. A further $600 \mathrm{ml}$. air was ${ }^{+}$ withdrawn, and because of the continued air leak a $T$ needle was inserted in the chest and connected to a $\underset{\mathbb{D}}{\circ}$ waterseal bottle.

The next day the condition of the child was very $\frac{\vec{D}}{\mathbb{Q}}$ poor and there was a large amount of air coming 


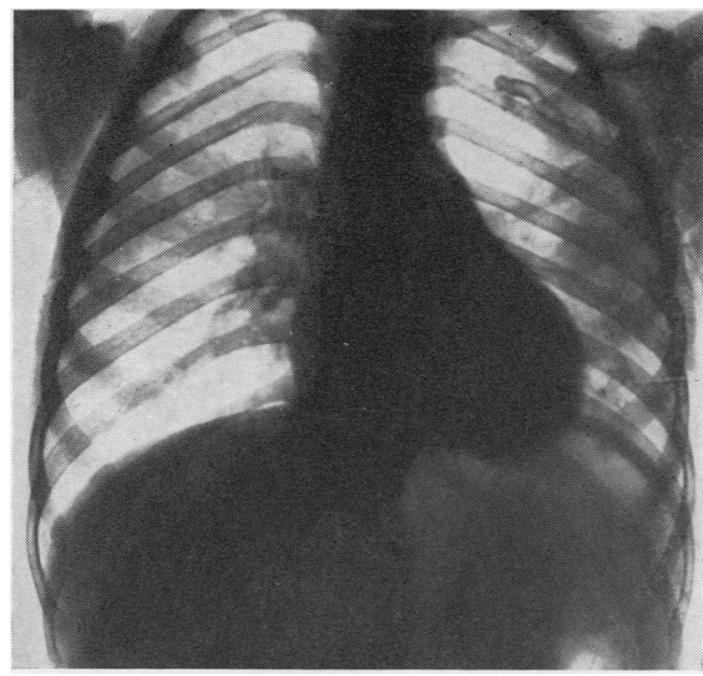

Fig. 7.-Case 3: Radiograph taken on January 15, 1952, three days after the accident.

from the left chest. A fractured bronchus was diagnosed and an intercostal drain inserted, with great improvement. On January 14 intrapleural penicillin was given and the tube retained. The intercostal tube was removed on January 18 ; the patient was still in the oxygen tent and too ill to bronchoscope. A chest radiograph on January 24 (Fig. 8) showed a left pneumothorax with an airless lung.

On January 26 bronchoscopy was performed, and half an inch down the main bronchus the lumen was seen to be completely occluded by granulation tissue. The left upper lobe orifice was not visible.

On January 27 a left pneumonectomy in the facedown position was performed. The chest was opened through the bed of the sixth rib; a pneumothorax was present and the lung was completely collapsed, solid, and palpable. The lung was only very lightly adherent to the chest wall in one or two places over the upper lobe. The pleural aspect of the intercostal drainage site was well healed. It was at once evident that there was extensive haematoma formation underneath the mediastinal pleura, which was intact apart from a small tear $\left(\frac{1}{2} \mathrm{~cm}\right.$.) overlying the upper surface of the pulmonary artery. No bronchial communication could be demonstrated at this stage. The mediastinal pleura was widely opened and the posterior surface of the bronchus exposed. During this dissection a tear in the back wall of the bronchus immediately above the upper lobe orifice and apparently extending into it was exposed. This rent was extended in a backward direction by a small incision and the interior of the bronchus inspected. It was now evident that the cartilaginous part of the bronchial wall had received considerable damage in addition to the tear in the membranous portion. At this point a considerable amount of yellow mucopus was expelled from the collapsed lung. The possibility

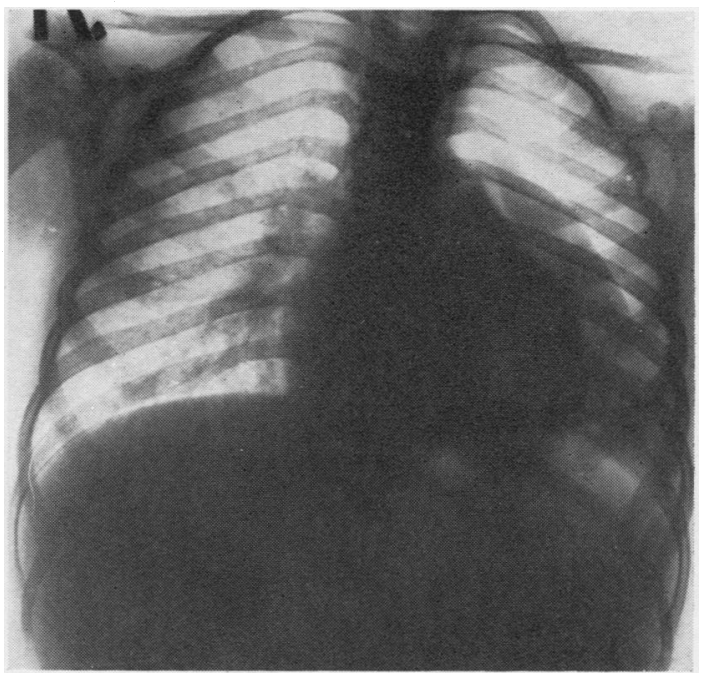

Fig. 8.-Case 3: Radiograph three days before left pneumonectomy.

of plastic repair with a view to saving the lung was carefully considered but rejected on account of the state of the lung and more particularly on account of the situation of the tear, extending as it did into the upper lobe bronchus. Left pneumonectomy was therefore carried out, the bronchus being amputated at the level of the bifurcation and closed with interrupted "mersilene" and the three pulmonary vessels individually ligated and divided. This procedure was carried out without incident and the chest was closed with three layers of continuous catgut, after insertion of penicillin into the pleural cavity.

The post-operative progress was uneventful and the patient was discharged on February 13, 1952. The child remains well.

CASE 4.-H. G., a girl aged 5 years, was run over by a car on July 6, 1953, the front and back wheels passing over the chest and abdomen. She was admitted to the North Middlesex Hospital under the care of M.B., where on examination she was found to have multiple abrasions of the arms and head. The pulse rate was 120 , respirations 36 , laboured, but she was not cyanosed. The trachea was to the right with cardiac dullness in the right chest, and there were signs of a left pneumothorax. There was epigastric tenderness. A chest radiograph confirmed a large left pneumothorax with displacement of the heart to the right and a fracture of the first right rib. A needle connected to an underwater seal was inserted in the left chest. On July 7 (Fig. 9) her general condition was much improved and the patient was breathing quietly. Next day the needle was removed and air taken off, leaving a high negative pressure in the left pleural cavity. The child was quite well and eating.

On July 9, 1953, bronchoscopy was performed under general anaesthesia. The left main bronchus 


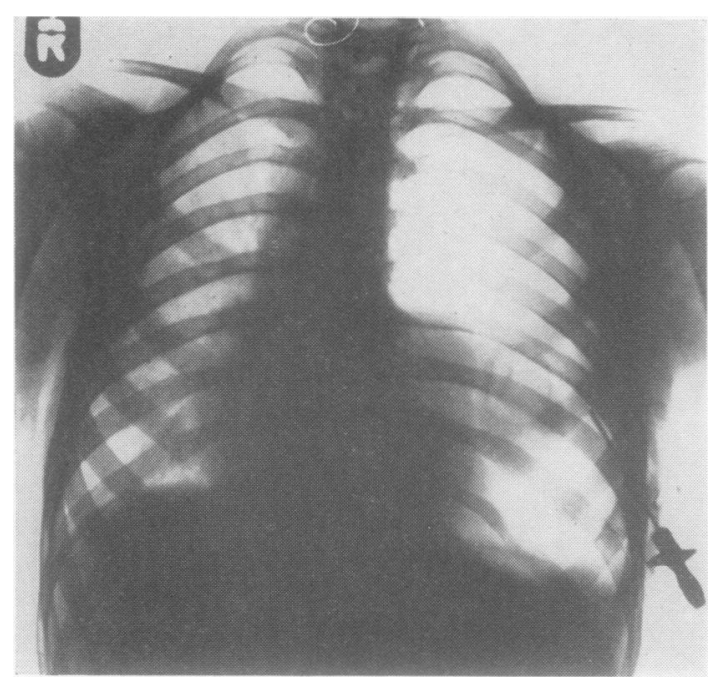

FiG. 9

was completely occluded by blood clot and granulation tissue, some of which was removed with biopsy forceps.

A week later a radiograph of the chest showed a complete atelectasis of the left lung with increasing mediastinal shift to the left (Fig. 10). On July 29

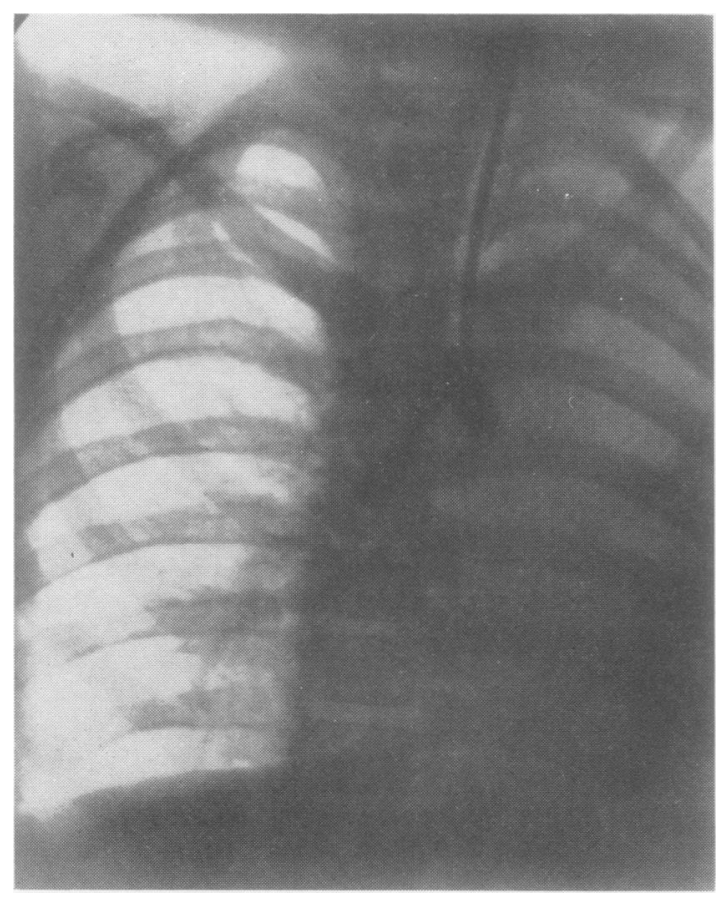

Fig. 11.-Case 4: Pre-operation bronchogram showing left bronchial obstruction.

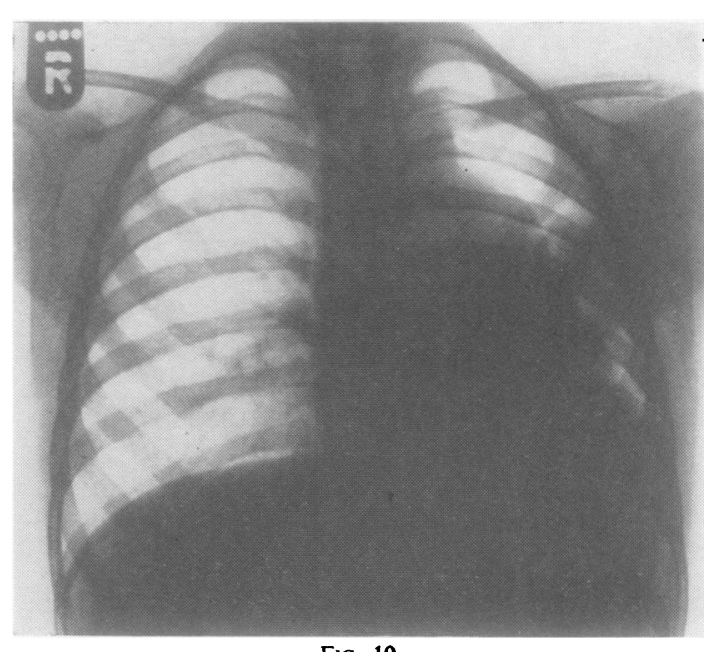

Fig. 10

FIG. 9.-Case 4: Radiograph 24 hours after the accident. Fig. 10.-Case 4: Radiograph two weeks after the accident.

bronchoscopy under general anaesthesia was repeated. $\vec{\oplus}$ There was still some granulation tissue in the left? main bronchus, which was removed, and then anV attempt was made to dilate the stricture in the bronchus with bougies. On September 15, 1953, a radiograph of the chest showed that there was still no reexpansion of the left lung, and there was further\% mediastinal shift to the left. The next day broncho- $\varrho$ scopy under general anaesthesia showed complete $\overrightarrow{\vec{O}}$ obstruction of the left main bronchus about half an 3 inch from the carina by apposition of the bronchial walls. A bougie could not be passed through this: obstruction. The child at this time was fit and free from symptoms, and further radiographs showed no change. On October 9, 1953, a bronchoscopy and bronchogram (Fig. 11) were done under general anaes- $x$ thesia and showed complete obstruction to the iodized oil about 1 in. from the carina. It was decided to explore the left chest with the object of repairing the injured bronchus if possible.

On October 13, 1953, with the patient in the lateralo position, the chest was opened through the fourth left $>$ intercostal space. The left lung was found to be음 atelectatic. It was retracted and the structures at the root of the lung were dissected out. A retractingo tape was passed round the pulmonary artery. The distal end of the left main bronchus was easily identified and found to be firmly healed over with fibrous $\omega$ tissue. It was seen that the main bronchus had been? fractured transversely just proximal to the origin ofo the left upper lobe bronchus. The proximal end of the main bronchus was found with difficulty under the? arch of the aorta, and it also was completely healed over with fibrous tissue. The two ends were abouto 2 in. apart, and there was no intervening tissue what $-\vec{D}$ ever. The proximal end was incised and opened, $\frac{\odot}{\mathscr{Q}}$ revealing the carina ; this end was temporarily closed with gauze packing. The distal end was next opened 
and thin mucopus escaped. This was sucked out and subsequently shown to be sterile on culture. After all the secretion had been removed from the lung by suction a catheter was passed into the upper and lower lobe bronchi and air injected. The left lung immediately expanded, appearing pink and healthy. The two ends of the bronchus were then approximated by interrupted fine stainless steel sutures, the knots being tied on the outside. The lung immediately aerated on inflation and the suture line appeared to be airtight. The chest was closed with a basal drain connected to a waterseal bottle.

A radiograph the next day showed partial aeration of the left lung, but a considerable pneumothorax was present. On October 15, two days after operation, the basal tube was taken out and air removed by a pneumothorax machine. Twenty-four hours later the patient was up and about, with the left lung almost fully expanded. On October 21 the sutures were removed, the wound healed, and the left lung was fully aerated. She was discharged to the convalescent home.

On April 7, 1954, bronchoscopy and a bronchogram (Fig. 12) were done under general anaesthesia. The left main bronchus was smooth and no sign of the suture line could be seen. There was no stenosis. The left bronchogram showed normal bronchi in all segments. The child's general condition was excellent and she remains well. Fig. 13 is a radiograph taken on April 27, 1954.

CASE 5.-C. P., a 5-year-old boy, fell from a moving van driven by his father on August 26, 1954 . The father said that the boy in his fall hit the edge of the pavement and for a short time was unconscious. $\mathrm{He}$ was admitted at once to the Great Yarmouth and

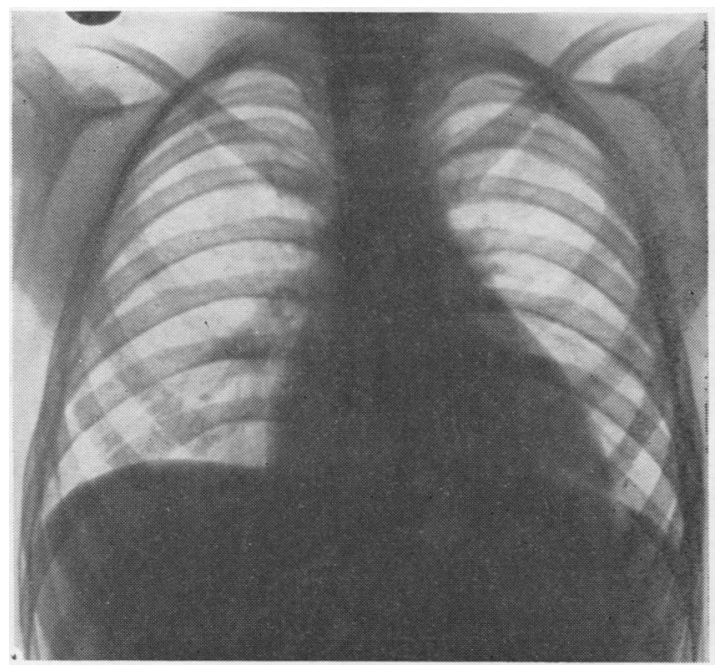

FIG 13

FIG. 13.-Case 4: Radiograph in April, 1954.

Fig. 14.-Case 5: Radiograph shortly after the accident.

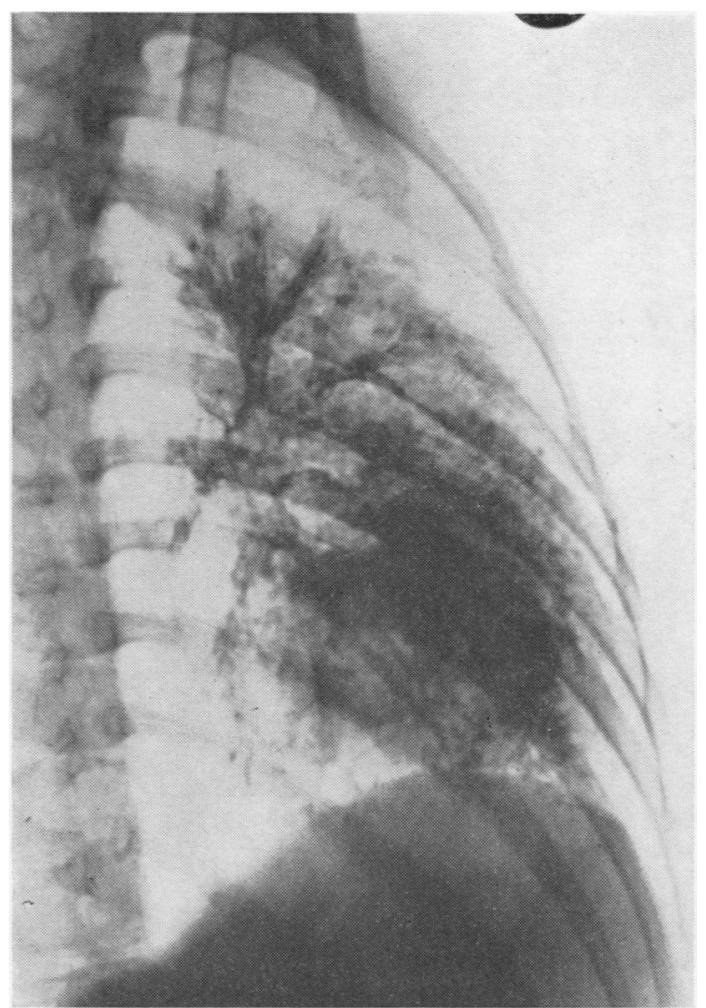

FIG. 12.-Case 4: Post-operative bronchogram in April, 1954, showing normal left bronchial tree.

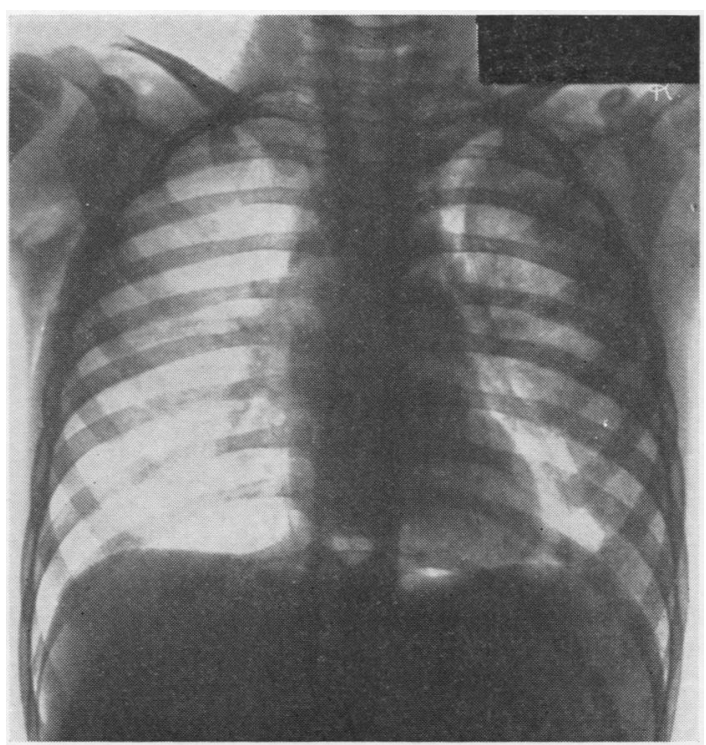

FIG. 14 


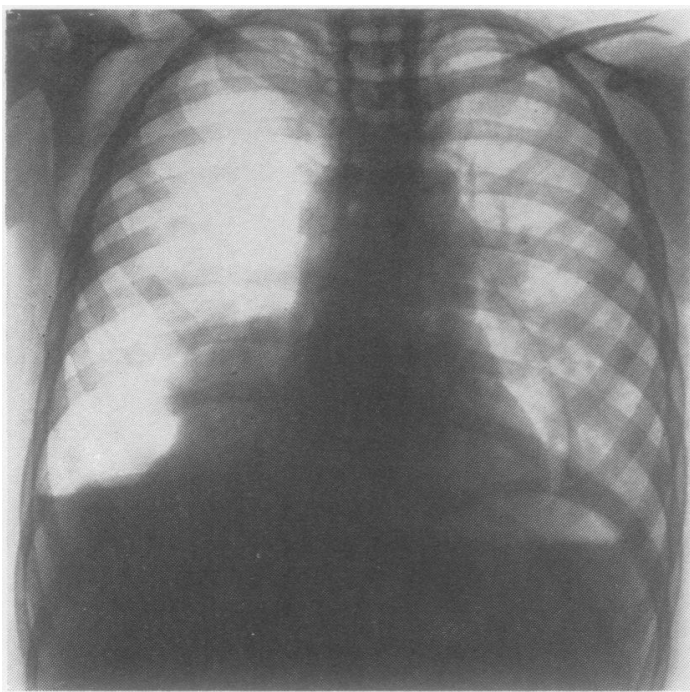

FIG. 15

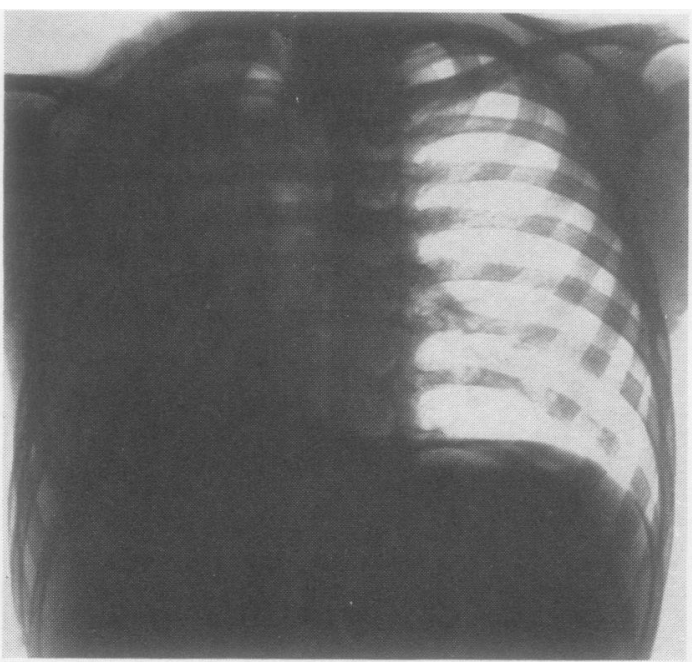

FIG. 16

FIG. 15.-Case 5: Radiograph 24 hours after the accident.

FIG. 16.-Case 5: Pre-operative radiograph in November, 1954. FIG. 17.-Case 5: Radiograph taken immediately after operation. FIG. 18.-Case 5: Radiograph nine months after operation.

Gorleston General Hospital under the care of Drs. I. M. Young and J. Salmon. The child was shocked, there were abrasions of the head, the pupils were unequal, and there was surgical emphysema in the neck. Because of the latter a chest radiograph was taken (Fig. 14). This showed a right pneumothorax, small opacities in the left lung, mediastinal emphysema and what was apparently a pneumopericardium. At a later date the radiologist pointed out that this was not a pneumopericardium but a stripping by air of the mediastinal pleura on both sides.

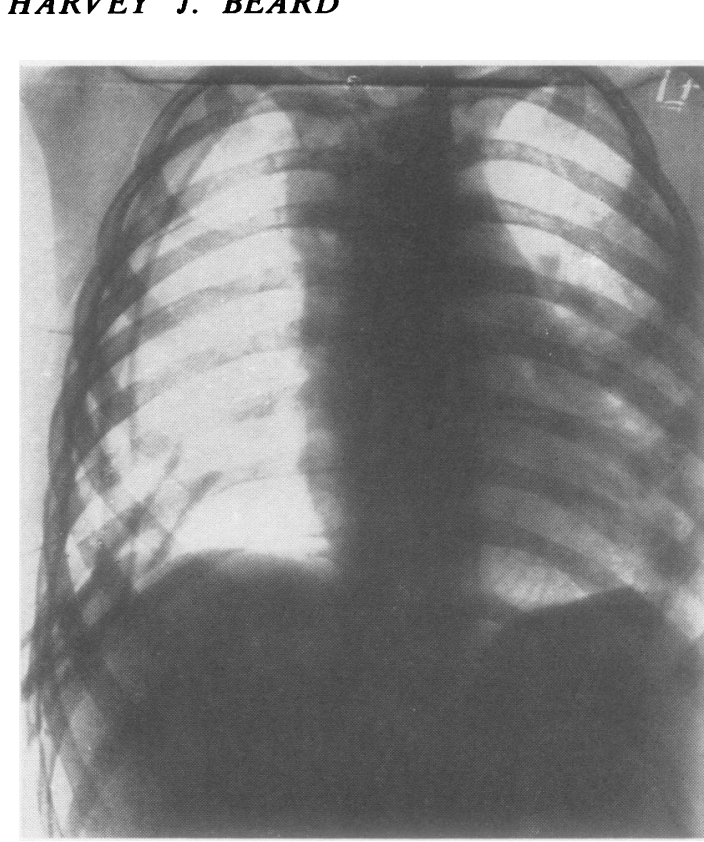

FIG. 17

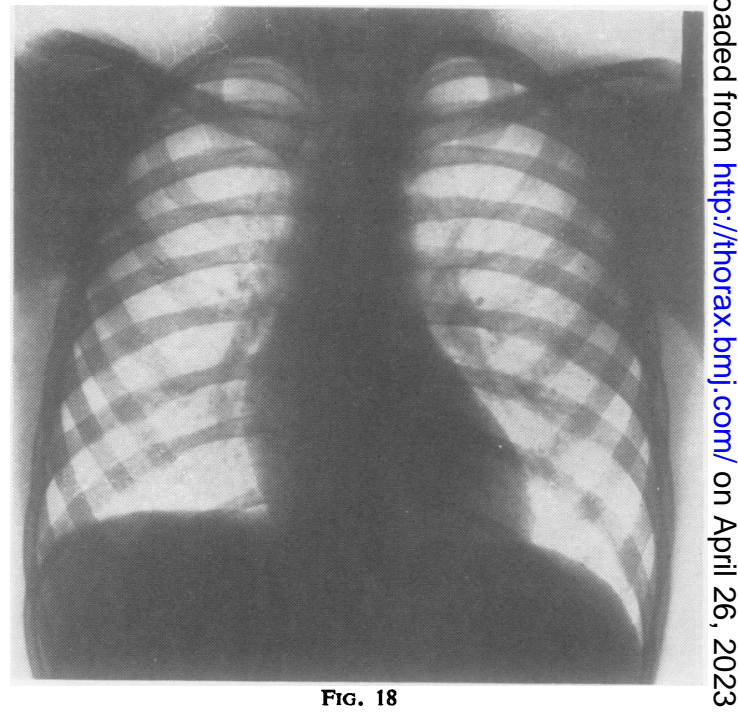

The day after the accident the pneumothorax was more obvious (Fig. 15), and a needle connected to an $\frac{\bar{\Phi}}{\bar{D}}$ underwater seal was inserted into the right chest. When signs of a tension pneumothorax appeared this needle was replaced by an intercostal drainage tube $\bar{O}$ and continuous suction. Twelve days after the acci- $\frac{\vec{C}}{\mathbb{Q}}$ dent a radiograph showed no pneumothorax but com- $\stackrel{\leftrightarrow}{\mathscr{Q}}$ plete atelectasis of the right lung. During this time $\_$ in hospital pleural fluid cultures were sterile, a Man- $\bar{\sigma}$ toux test had proved positive, and a radiograph of $?$ the skull showed no evidence of fracture. 
On September 10, 1954, the child was transferred to the Department of Thoracic Surgery, Kelling Hospital, under the care of H.J. B. The intercostal tube was removed, and the next day under general anaesthesia the child was bronchoscoped. Immediately distal to the main carina the entrance to the right main bronchus was ragged-looking and completely blocked. On the medial wall there seemed to be a piece of cartilage pushing inwards. After repeated suction some pieces of mucus and old blood came away, revealing a small opening. Appearances were so distorted that it was not possible to say if this opening was into the main bronchus or not. However, a radiograph taken immediately afterwards showed an almost completely aerated lung.

This promising state of affairs continued, although on deep inspiration the child's chest produced a most curious noise, presumably due to the organ pipe effect of the bronchus, across which at bronchoscopy had been seen stretched a sort of reed of mucous membrane. There was no great confidence about the future of this lung, but it seemed justifiable to wait and see what happened. He returned to his original hospital on September 15.

The child remained in good health until the end of October, some eight weeks after the accident, when the weekly radiograph again showed complete atelectasis of the right lung.

He was readmitted to Kelling Hospital on November 1, 1954, with radiographic appearances as in Fig. 16. On two occasions he was bronchoscoped under general anaesthesia, with vain efforts on the second occasion to pass dilators. There was a complete block of the right main bronchus just distal to the main carina.

On November 18, 1954, 12 weeks after the accident. a right thoracotomy was performed with the patient in the lateral position. The lung was found to be atelectatic. Approach to the hilum was difficult because of extensive fibrosis. The pulmonary artery was isolated first and the stenosed area of bronchus gradually exposed. The main bronchus appeared to be replaced by a fibrous cord over a distance of approximately $1 \frac{1}{2} \mathrm{~cm}$. immediately distal to the main carina. The main bronchus was cut across distal to the stenosed area, and through the now open bronchus a small quantity of whitish thick secretion was removed by suction from the collapsed lung. Using an ordinary $20 \mathrm{ml}$. syringe and small catheter it was next verified that the lung would inflate. The proximal end of the stenosis was followed up to the main carina and cut a cross twice before a normalsized opening was found, which was seen to be exactly at the carina. The tracheal opening was intermittently closed with Allis's forceps to help the anaesthetist (Dr. A. I. Ward). The distal end of the bronchus was trimmed to match in size the proximal opening. While this was being done some slight bleeding of the bronchial wall was seen for the first time; the normal bronchial blood supply had been destroyed by the accident. No further trimming could have been done without encroaching on the upper lobe bronchus. The two bronchial ends were then anastomosed by six interrupted No. 4 nylon sutures.

The lung was gently inflated and expanded well. Two further bronchial sutures controlled remaining air leaks. No attempt was made to cover the suture line with a pleural flap. Upper and lower drainage tubes were inserted, and a radiograph (Fig. 17) taken on the table at the end of operation showed an almost completely re-expanded lung. The lung remained fully expanded and convalescence was rapid.

Bronchoscopy nine months after the operation showed a slightly narrowed entrance to the right main bronchus. This was clearly shown in a tomograph taken at this time. A straight radiograph (Fig. 18) shows a normal chest. The child is healthy, but the pupils remain unequal.

CASE 6.-This is not a traumatic bronchial rupture in the same sense as the other five cases, but is of interest, as the events leading to the bronchial injury are commonplace.

The patient, B. B., a boy aged 2, "choked on a bread crumb." He was admitted on November 2, 1955 , to the Children's Department of the Borough General Hospital, Ipswich, under the care of Dr. R. M. Mayon-White. The child had marked surgical emphysema of the neck. A radiograph of the chest on the day of admission (Fig. 19) showed extensive mediastinal emphysema extending up into the neck and elevation of the mediastinal pleura, which was more obvious on the left side. The horizontal shadow at the left costophrenic angle suggested a small collection of fluid, and was the only evidence that a pneumothorax might be present. In the second radiograph (Fig. 20) nine days later, the mediastinum is displaced to the right by an obstructive emphysema

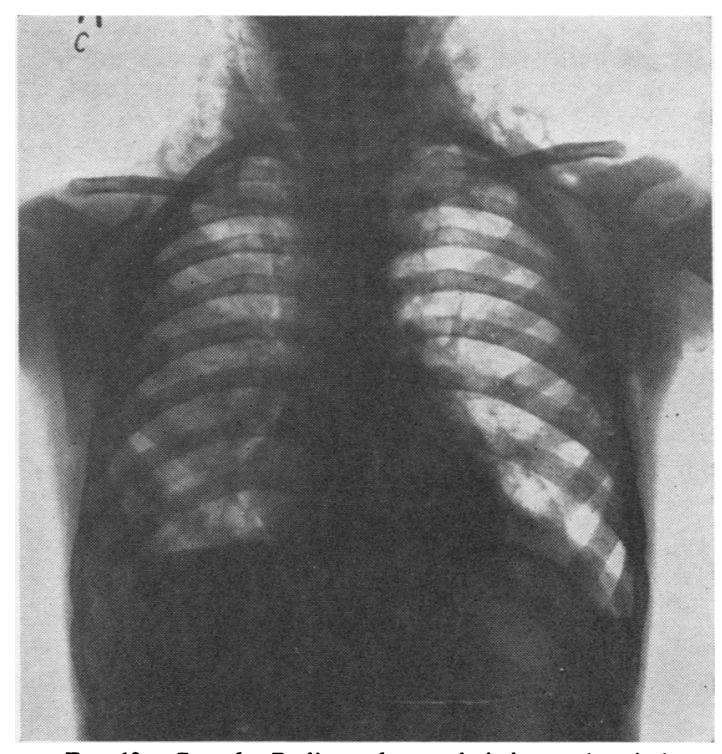

FIG. 19.-Case 6: Radiograph on admission to hospital. 


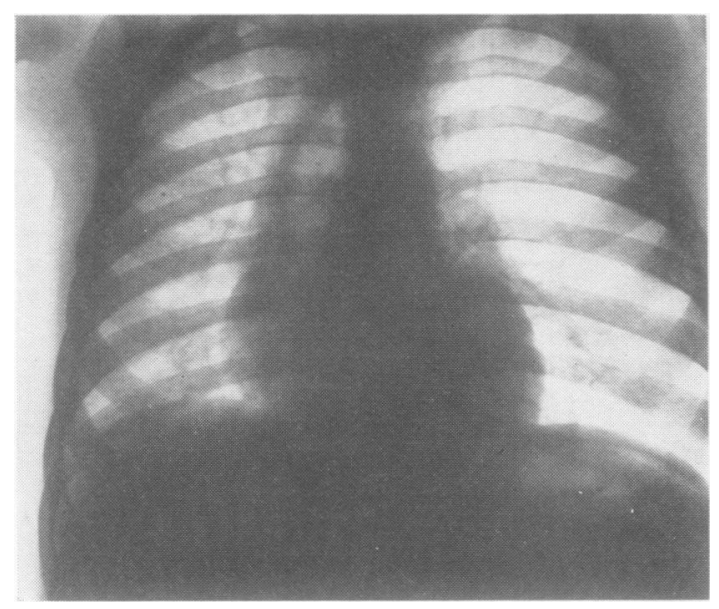

FIG. 20.-Case 6: Radiograph nine days after admission.

of the left lung, which in the radiograph (Fig. 21) of November 25 has given place to atelectasis of the left upper lobe with the mediastinum now slightly to the left. Later radiographs again show an obstructive empyhsema of the left lung with mediastinal displacement to the right. A later series of radiographs suggest that the obstructive emphysema is slowly resolving.

During his first days in hospital the child received penicillin by injection, and throughout his hospital stay he was treated by postural drainage and thoracic physiotherapy. For a time he had a low-grade pyrexia. The general condition steadily improved. Early in December, 1955, he was transferred to the convalescent home at Cromer, where the physician, Dr. R. B. Fawkes, showed the films to H. J. B. in consultation.

The mediastinal emphysema and subsequent behaviour of the left lung prove this to have been a left bronchial injury, presumably involving the upper lobe bronchus, and the bronchus could be visualized passing through its phases of partial to complete and back to partial obstruction.

As the child was not seriously ill and as a spontaneous recovery seemed possible, a conservative policy of treatment was adopted and bronchoscopy was not performed. Six months after the original incident a radiograph showed an apparently healthy chest.

\section{Discussion}

The paper by Kinsella and Johnsrud (1947) still stands as the classical work on traumatic rupture of the bronchus even though the passage of time has modified some of their suggestions for treatment.

The six cases reported here were all children, and the injury is generally considered to be more

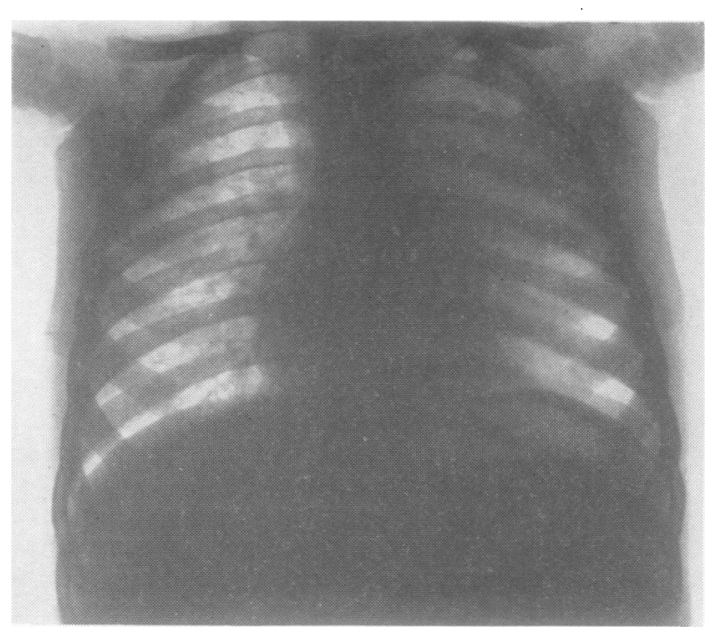

FIG. 21.-Case 6: Radiograph taken three weeks after admission to hospital.

likely in children because their supple chest wall allows external forces to be transmitted more easily to the trachea and bronchi than is the case in the adult. However, it is worth pointing out that well over half the 38 cases of Kinsella and Johnsrud were adults. Others also have reported adult cases (Weisel and Jake, 1953; Griffith, 1949 ; Paulson, two cases, 1951 ; Fowler, 1955).

The site of the rupture is near the main carina and can involve the trachea itself or extend into the upper lobe bronchi. In this injury there is usually a history of being run over by a vehicle, the wheels actually passing over the victim as in Cases 1, 2, and 4. In Case 3 a heavy object fell on the child. Case 5 was due to the child falling from a moving vehicle, and the only similar case found was one of those collected by Kinsella and Johnsrud where a jockey fell from his horse, although in the case reported by Dark and Jewsbury (1955) the child simply fell off a wall. How the bronchus came to be injured in Case 6 is a matter for speculation. All that is known for certain is the child had a choking fit after inhaling a bread crumb. When this happens to a child any adult present will almost invariably proceed to thump it heartily on the back between the scapulae, and one can postulate that an extra hard thump at the time of a firmly closed glottis and high intrabronchial pressure could cause disruption of the bronchus.

There are often concomitant injuries from the severe vehicular accidents, as the head injuries in Cases 1, 3, 4, and 5, with a fractured femur also in Case 3. These other injuries may at first overshadow the chest injury and any of them be suffi- 
cient to cause death, and indeed anxiety caused by these multiple injuries may be enough to prevent early thoracotomy for the bronchial injury : this was so in Cases 3 and 5.

Only in Cases 1 and 4 was there an associated single rib fracture, but as all the cases were children that might account for the absence of fractured ribs. Review of the literature reveals that children often sustain no rib fracture whereas in adults fractured ribs are usually accompaniments of a bronchial injury. This is of importance in diagnosis, for in adults it may be thought that the fractured ribs are the sole cause of the surgical emphysema and pneumothorax. The more serious injury to the bronchus is then overlooked. It is interesting to note that in a few cases, adults and children, the first rib only has been fractured, as in Case 4.

Mediastinal emphysema, palpable in the suprasternal notch and neck in Cases 5 and 6, should always be regarded as evidence of rupture of the tracheal, bronchial or, more rarely, oesophageal wall. In Case 5 it was the mediastinal emphysema which lifted the mediastinal pleura and created a radiographic appearance resembling a pneumopericardium; there was a similar appearance in the first radiograph of Case 6 .

In most cases a radiograph, as well as showing mediastinal emphysema, will show a pneumothorax, but this is not an essential feature. Kinsella and Johnsrud state that it developed in 16 out of 38 cases. Pneumothorax is not mentioned in the records available of Case 2 and there is some doubt if one existed in Case 6 , but one was present in the other four cases. The pneumothorax varied from a small one in Case 5 to a bilateral one with tension pneumothorax on the left side in Case 3 ; the latter case is also an example of the continued large air leak which may follow bronchial rupture.

Haemoptysis is not a necessary sign of a ruptured bronchus; it is infrequent in reported cases, and was not present in those here reported. It is in fact remarkable how infrequently serious haemorrhage occurs with this injury. A mediastinal haematoma in the region of the tear is likely, and at operation in Case 3 was found to be extensive, the haemorrhage presumably coming from torn bronchial vessels. Even if bleeding into the bronchus is only slight it may easily, in the unconscious patient, enter the other lung and cause areas of atelectasis which were probably the cause of the left lung shadows in the first radiograph of Case 5 . Such developing areas of atelectasis, or, it may be, pulmonary oedema, can increase the respiratory difficulties. Reference to the radiographs of Cases 3, 4, and 5 will show that intrapleural bleeding was negligible, and study of several reported cases shows a similar absence of intrapleural haemorrhage. This would be expected because traumatic rupture of a bronchus is primarily a mediastinal and subpleural occurrence. This radiological picture is so consistent that the presence of any considerable amount of blood in the pleural cavity soon after the accident will probably mean that the lung has been lacerated. Severance of the main pulmonary vessels at the hilum is only of theoretical interest as death is almost immediate, but despite their proximity to the torn bronchus these vessels often escape injury.

Treatment of the chest condition only will be considered here. Fowler (1955) has said that where there is a bronchial rupture an expectant policy can be disastrous, and he suggests that diagnostic needling with underwater seal be done in all cases of non-penetrating chest injury with pneumothorax. This needling will relieve a tension pneumothorax and show if air is continuously escaping from the bronchial tree. When tension pneumothorax is diagnosed the prompt insertion of any wide-bore needle through a rib interspace high up in front can be life-saving, as was so in the case reported by Scannell (1951). In Cases 3, 4 , and 5 an intrapleural needle connected to an underwater seal was introduced early, but in Cases 3 and 5 was later replaced by an intercostal tube to cope with the continuous air leak.

When the patient has been tided over the early stages of shock and respiratory difficulties, the next step is to try and assess the extent of the damage and decide on the further treatment.

In any patient with a suspected bronchial injury bronchoscopy should be performed, although the reasons for not doing so in Case 6 have been given. By means of the bronchoscopy, after the removal by suction of any obtruding clot, the proximal site of the injury can be accurately gauged, but to assess the extent of the injury beyond this point is much more difficult. At bronchoscopy in Cases 3, 4, and 5 evidence of serious injury was seen-blood clots, granulation tissue, torn pieces of mucous membrane, and in Case 5 a protruding piece of cartilage-but whether the injury was a linear tear or a partial or a complete severance of a bronchus it was impossible to tell. During the first few days after the accident bronchoscopy should not be undertaken unless the means are at hand for a thoracotomy. The bronchoscopic suction could dislodge 
blood clot which has temporarily closed the bronchial rupture, and in some circumstances uncontrollable tension pneumothorax might supervene and require immediate operation. It is worth persevering with the bronchial toilet, for an airway may be re-established. In Case 5, for example, it was 20 minutes before the bronchial obstruction was cleared during bronchoscopy on the fifteenth day after the accident. The reexpansion of the lung which followed lasted only for about six weeks, but this interval enabled the child to regain good health, which probably helped him through the subsequent operation. In the second personal case of Kinsella and Johnsrud there was after bronchoscopy on the fourteenth day similar temporary re-aeration of the lung, followed later by complete atelectasis, for which operation at that time was not advised.

If the injury to the bronchus results in a stricture an attempt will probably be made to dilate this with bougies, but in the literature studied there was no record of permanent success after this manœuvre, and similarly in Case 1 above there was only temporary success. In strictures of this severity it would seem that the constricting fibrous tissue will always win.

Cases of bronchial rupture to be treated by open operation fall into two groups, the first where operation must be undertaken as early as possible in order to save life. Such were the cases of Dark and Jewsbury (1955), operated on 24 hours, and of Scannell (1951), operated on three hours, after the accident ; in the latter the child's condition was so grave that an anterior thoracotomy was necessary. In cases as urgent as these preliminary bronchoscopy cannot be performed. The repair of the torn bronchus may be possible in this group or it may mean resection of the whole lung, as was necessary in Case 3. This case comes into this group, for it had been decided that early operation was essential. However, the child was so desperately ill that the surgeon dared not remove him from the oxygen tent for several days. Having a bilateral pneumothorax and fractures of both clavicle and femur, this case well illustrates the devastating effects of multiple injuries added to that of the fractured bronchus.

The second group of cases are those where a more leisurely approach can be undertaken. Operation in this group will nearly always be for an atelectatic lung, although in the case reported by Griffith (1949) the condition was one of obstructive emphysema, and obstructive emphysema was a feature in two phases of Case 6 . Cases 4 and 5 were in this group, and in each there was atelectasis of the whole lung which had been present for some weeks. In such cases a decision has to be taken whether to leave in situ a permanently atelectatic lung or perform a thoracotomy to attempt a bronchial repair and if that is impossible remove the lung.

When Kinsella and Johnsrud wrote their paper in 1947 they suggested measures for relieving the $\vec{\omega}$ discomfort which might be associated with complete atelectasis. We believe, with the experience $x$

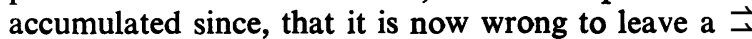
permanently collapsed lung in the chest, as there will always be a risk of infection and impaired respiratory function.

Occasionally patients will be seen who have had a collapsed lung for many years. Cases 1 and $2 \vec{\square}$ are examples. Case 1 has a completely closed $\mathbb{D}$ bronchus and atelectatic lung; the latter condi- $\Phi$ tion may well have been present for 20 years. In $\overline{0}$ Case 2 the lung may have been collapsed for $\mathbb{Q}$ 10 years. The bronchoscopic findings in this $\overrightarrow{0}$ patient suggested that a small bronchial opening of still existed, although the bronchogram showed an occluded bronchus. It is probably unwise to operate on these patients with long-standing atelectasis, unless signs of infection in the collapsed lung with deteriorating health call for $a \stackrel{\circ}{\Phi}$ pneumonectomy.

When operation is decided upon it may be $\overrightarrow{\overrightarrow{0}}$ impossible to say beforehand whether or not the bronchus is completely torn across. In Case 5 F complete rupture was unlikely, as aerated lung was seen in the radiograph after the first broncho- $\overrightarrow{0}$ scopy, and in Case 4 an attempt had been made to pass bougies through what was thought might $\stackrel{x}{\circ}$ be a stricture, whereas subsequent operation 3 showed a complete rupture.

It is still uncommon to find cases of traumatic bronchial rupture treated by repair with preserva- 0 tion of the whole lung, but this was possible in Cases 4 and 5. The vagaries of the tear decide if the whole lung can be saved or not. On the right side the tear is more likely to extend into the $O$ upper lobe bronchus, which may necessitate lobec- $N$ tomy at operation. On the left side there is less $\mathrm{N}$ risk of the tear extending into the upper lobe, $O$ although it did so in Case 3 and probably also in Case 6, but the technical difficulties of suturing a torn main bronchus are greater on the left side. $\stackrel{\mathcal{D}}{+}$

When the chest is opened the cause of the 0 atelectasis is frequently found to be a stricture of 0 the bronchus. Griffith (1949) resected a stricture $\stackrel{\mathbb{D}}{\circ}$ but left intact a portion of the membranous wall $\mathbb{D}$ with its bronchial blood vessel. End-to-end suture $\frac{a}{\sigma}$ was used. Paulson (1951) in his second case in- 
cised a stricture of the right main bronchus, and to repair the defect left in the bronchus and lower part of the trachea used a dermal graft. When operating on Case 5 there was no hesitation in excising the whole of the stenosed area of bronchus, as it appeared that this could be accomplished without encroachment on the trachea or the upper lobe bronchus. However, at operation, instead of a stricture a completely severed bronchus may be found with the ends widely separated. Such was Case 4. A similar case was that of Weisel and Jake (1953). Their patient was an adult aged 32, and the lung had been collapsed for over seven weeks after the accident of complete bronchial rupture and destruction of bronchial vessels. After decortication the lung inflated and the right main bronchus was implanted into a window made in the side of the trachea.

For long it was believed that an intact bronchial blood supply was essential for bronchial repair, but there have now been many reports proving that complete interruption of the bronchial vessels is no bar to the healing of a bronchial anastomosis. Recently Jackson, Jones, and Sellors (1955) have reported the successful excision of tuberculous strictures with end-to-end anastomosis. They state that the bronchial artery supply is interrupted by the division of the bronchus, but this does not appear to jeopardize the vitality of the junction. In Cases 4 and 5 the continuity of the bronchial vessels had been completely interrupted for a period of over three months, yet with simple endto-end suture healing was satisfactory, and in neither case was there any attempt to cover the suture line with pleural flaps. In Case 4 it is worth noting that both bronchial ends were completely healed over with fibrous tissue, were 2 in. apart, and had no intervening tissue whatever. In Case 5 the stricture appeared to be just a fibrous cord without a lumen, and this when cut across did not bleed at all. Both these cases at operation revealed an atelectatic lung completely cut off from the main bronchus; in one instance it had been so for three months and in the other for five weeks, yet after removal of bronchial secretions these lungs were easily inflated without decortication. The bronchial secretions in Case 4 were proved sterile. These two cases demonstrate conclusively that a lung with a severed or completely closed main bronchus and devoid of its bronchial blood supply is, following operative repair, capable of re-aeration and full function, even after being completely atelectatic for weeks or months.

In conclusion, the authors believe that, when a patient has a traumatic rupture of the bronchus for which operation is indicated, then this operation should be performed early. If this is not practicable and atelectasis of the lung, the most common sequel, supervenes, then open operation for repair of the bronchus should be undertaken, even though the atelectasis is of some months' duration.

\section{SUMmARY}

Six cases of traumatic rupture of the bronchus are reported and commented upon, together with a review of some of the literature.

Early operation is advocated if possible, but late operation should be undertaken in preference to leaving a permanently atelectatic lung in the chest.

Two of the reported six cases had late operative repair of the torn bronchus with end-to-end anastomosis, resulting in full re-expansion of the lung after atelectasis of prolonged duration.

We are indebted to Mr. O. S. Tubbs, Mr. R. Abbey Smith, Mr. Gordon Cruickshank, and Dr. R. M. Mayon-White for details of Cases 1, 2, 3, and 6, to Mr. E. C. Wynne-Edwards for help with Case 5, and to all the physicians mentioned.

\section{REFERENCES}

Dark, J., and Jewsbury, P. (1955). Thorax, 10, 62.

Fowler, A. W. (1955). Brit. med. J., 1, 85.

Griffith, J. L. (1949). Thorax, 4, 105.

Jackson, J. W., Jones, P. H., and Sellors, T. H. (1955). Ibid., 10, 229.

Kinsella, T. J., and Johnsrud, L. W. (1947). J. thorac. Surg., 16, 571. Paulson, D. L. (1951). Ibid., 22, 636.

Scannell, J. G. (1951). Ann. Surg., 133, 127.

Weisel, W., and Jake, R. J. (1953). Ibid., 137, 220. 